\title{
Diplopia Following Spine Surgery: A Case Series
}

\author{
Alaa A. Abd-Elsayed ${ }^{1,2}$, Wael Barsoum ${ }^{3}$, Gordon Bell ${ }^{4}$, Maged guirguis ${ }^{5}$ and Ehab Farag*,5 \\ ${ }^{1}$ Department of Anesthesiology, University of Cincinnati, Cincinnati, Ohio, USA \\ ${ }^{2}$ Department of Outcomes Research, Anesthesiology, Cleveland Clinic, Cleveland, Ohio USA \\ ${ }^{3}$ Department of Orthopedic Surgery, Cleveland Clinic, Cleveland, Ohio, USA \\ ${ }^{4}$ Department of Spinal Surgery, Cleveland Clinic, Cleveland, Ohio, USA \\ ${ }^{5}$ Department of Anesthesiology, Cleveland Clinic, Cleveland, Ohio, USA
}

\begin{abstract}
Introduction: Cranial traction leading to 6th nerve palsy is the main cause of developing diplopia following spinal surgery. Very few cases of abducens nerve palsy as a complication of surgery without cranial traction have been described. We review a series of four patients who developed diplopia following spinal surgery only one of which had cranial pins or apparent traction on the abducens nerve.

Case presentations: Case 1, a 14-year-old girl who had a posterior segmental instrumentation correction and fusion under general anesthesia in the prone position. Case 2, a 34 year old woman who had posterior fusion and fixation. Case 3, a 67 year-old woman who had a C5-C6, C6-C7 anterior cervical diskectomy and fusion procedure. Cranial traction was utilized. Case 4, an 18 year-old woman who had an anterior interbody spinal fusion. All cases developed facial edema and diplopia on the first day postoperatively.

Conclusion: Diplopia is a rare complication following spine surgery. It needs special attention as it can be attributed to several reasons. Facial edema, face traction and CSF leakage were the possible reasons in our cases. Diplopia was temporary and resolved spontaneously in all our cases.
\end{abstract}

Keywords: Diplopia, spine, fascial edema.

\section{INTRODUCTION}

Cranial nerve deficits have been described in the spinal surgical fields after trauma and spinal surgery with cranial traction [1]. It is theorized that traction forces can cause localized ischemia or changes in the position of the nerve. Cranial nerves found to be at risk include the accessory nerve [2], hypoglossal nerve [3], abducens nerve [1], glossopharyngeal nerve, and vagus nerve [4]. The abducens (sixth cranial) nerve is more often involved in causing neurological deficits [4]. There have been few reports of abducens nerve palsy as a complication of surgery without cranial traction [5]. Majority of cases were due abducens nerve palsy though diplopia can occur due to oculomotor and rarely to the trochlear nerve involvement. We describe a series of four patients who developed diplopia following spinal surgery.

\section{CASES PRESENTATIONS}

\section{Case 1}

A 14-year-old girl who presented to the clinic with a progressive idiopathic scoliosis. The patient had a posterior segmental instrumentation correction and fusion in under general anesthesia; the patient was operated in the prone position. She was transferred to the recovery room after

*Address correspondence to this author at the General Anesthesiology, Cleveland Clinic, 9500 Euclid Ave., Cleveland, OH 44195, USA; Tel: (216) 444-7550; Fax: 216-444-2294; E-mail: FARAGE@ccf.org surgery in stable condition. The patient developed facial edema and diplopia in the first day postoperatively. The diplopia resolved on its own within few days. Patient did not recall if he was seen by an ophthalmologist neither recalled the actual duration of the diplopia. Management details including duration of surgery and fluid administration for all cases is shown in Table $\mathbf{1}$.

\section{Case 2}

A 34-year-old woman presented to the clinic with post laminectomy kyphosis.

She had posterior fusion and fixation. The patient was operated in the prone position. Patient developed facial edema and diplopia on the first postoperative day. Patient was seen by the ophthalmologist who excluded any organic cause for developing the diplopia. Diplopia resolved on its own by post-operative day No. 5 .

\section{Case 3}

A 67-year-old woman with history of uncontrolled diabetes mellitus presented with progressively worsening balance, difficulty walking, neck and back pain, and upper- and lower-extremity weakness. She had a C5-C6, C6-C7 anterior cervical diskectomy and fusion procedure. Head pins were used. The patient was operated in the supine position. The patient was extubated from the operating room and she was then moved to the OR stretcher in stable condition, and then was transferred to the Recovery Room. Patient developed 
Table 1. Management Details Including Duration of Surgery and Fluid Administration

\begin{tabular}{|c|c|c|c|c|c|c|c|c|c|c|c|c|c|c|c|c|c|c|c|}
\hline שू & 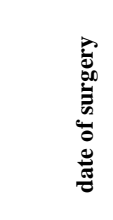 & 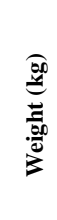 & 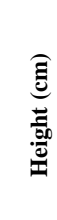 & 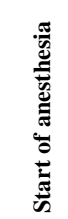 & 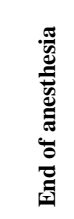 & 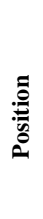 & 密 & 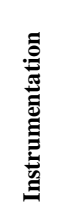 & $\frac{n}{0}$ & 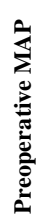 & 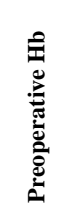 & 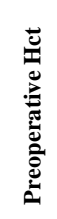 & 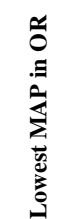 & 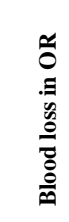 & 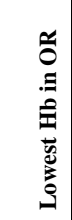 & 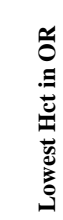 & 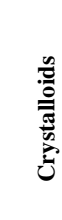 & $\begin{array}{l}\frac{n}{0} \\
\overline{0} \\
0\end{array}$ & 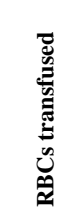 \\
\hline 2 & $8 / 17 / 2006$ & 45 & 147 & $7: 24$ & $19: 56$ & 0 & 34 & No & 2 & 77 & 11.1 & 35.3 & 57 & 3000 & 7 & 23 & 7000 & 1465 & 1000 \\
\hline 3 & $1 / 21 / 2008$ & 61.9 & 160 & 13:05 & $19: 12$ & 5 & 67 & Yes & 1 & 75 & 12.1 & 36 & 51 & 100 & 9.1 & 26 & 2350 & 1000 & 380 \\
\hline 4 & $1 / 30 / 2006$ & 48.9 & 160 & $7: 50$ & $17: 01$ & 1 & 18 & Yes & 2 & 66 & 13.5 & 41.6 & 50 & 500 & 10.9 & 23.1 & 2700 & 0 & 0 \\
\hline
\end{tabular}

facial edema and diplopia in the first postoperative day which resolved on its own within few days.

\section{Case 4}

An 18-year-old woman presented to the clinic with progressive thoraco-lumbar curve. She had an anterior interbody spinal fusion. The patient was operated in the left lateral decubitus position. The patient was then taken from the operating to the recovery room in satisfactory condition and she developed facial edema and diplopia in the first postoperative day. Patient was seen by the ophthalmologist who excluded the presence of any lesion that might cause this diplopia. The diplopia resolved on its own in post-operative day 3 on average as recalled by the patient.

\section{DISCUSSION}

Abducens nerve palsy, which causes diplopia without any other neurological signs, is reported to be the most common cranial nerve palsy [4]. Abducens nerve palsy has been reported to be a complication of several procedures including lumbar puncture, shunt operation, maxillary osteotomy, cranial trauma, and skull traction [1, 5-8].

The sixth, ninth, tenth, and twelfth cranial nerves have vertical or oblique course in the cranium making them the most vulnerable nerves to stretch injuries [9] with the abducens nerve being the most at risk of injury [1]. Barsoum et al. reported a case of apparent abducens nerve palsy after only $5 \mathrm{lb}$ of traction at lumbar spinal surgery [1]. The cause of the palsy was thought to be stretch of the cranial nerve secondary to traction using Gardner Wells tongs.

Abducens nerve palsy has been also recognized as a complication of lumbar puncture [8]. The likely etiology has been cited as caudal displacement and neurotoxic spinal arachidonitis. In our series, skull traction was used in only case
No. 3. Diplopia in Case No. 3 can be attributed to the traction forces while for case No. 2 fluid overload can be the reason as the patient received large amounts of fluids that might lead to edema and abducens nerve stretch. In the other 2 cases we believe that CSF leakage at surgery may be the cause of the palsy. One final etiology may be fluid overload causing a traction injury of the $6^{\text {th }}$ cranial nerve leading to diplopia as facial edema was detected in all cases postoperatively.

\section{CONCLUSION}

Diplopia is a rare complication following spine surgery. It needs special attention as it can be attributed to several reasons. Facial edema, face traction and CSF leakage were the possible reasons in our cases. Diplopia was temporary and resolved spontaneously in all our cases.

\section{REFERENCES}

[1] Barsoum WK, Mayerson J, Bell GR. Cranial nerve palsy as a complication of operative traction. Spine 1999; 24: 585-6.

[2] Dellon AL, Campbell JN, Cornblath D. Stretch palsy of the spinal accessory nerve: case report. J Neurosurg 1990; 72: 500-2.

[3] Kacker A, Komisar A, Kakani RS, et al. Tongue paralysis following head trauma. J Laryngol Otol 1995; 109: 770-1.

[4] Wilkins C, MacEwen GD. Cranial nerve injury from halo traction. Clin Orthop 1977; 126: 106-10.

[5] Nakagawa H, Kamimura M, Uchiyama S, Takahara K, Itsubo T, Miyasaka T. Abducens nerve palsy as a rare complication of spinal surgery: a case report. J Orthop Sci 2003; 8(6): 869-71.

[6] Hodgson AR. Halo-pelvic traction in scoliosis. Isr J Med Sci 1973; 9: 767-70.

[7] Jain KK. Aberrant roots of the abducens nerve. J Neurosurg 1964; 21: 349-51.

[8] Miller EA, Savino PJ, Schatz NJ. Bilateral sixth-nerve palsy: a rare complication of water-soluble contrast myelography. Arch Ophthalmol 1983; 100: 603-4.

[9] Botte MJ, Byrne TP, Abrams RA, et al. Halo skeletal fixation: techniques of application and prevention of complications. J Am Acad Orthop Surg 1996; 4: 44-53. 